\title{
Measure of reflection and transmission coefficients for bone tissues
}

\author{
Y.RATNA KUMAR ${ }^{1}$, PROF. G.S.N. RAJU ${ }^{2}$ \\ Asst.Professor(C), Dept. of Electronics and Communication Engineering, College of Engineering (A), \\ Andhra University, Visakhapatnam, Andhra Pradesh, India ${ }^{1}$ \\ Vice-Chancellor, Andhra University, Andhra Pradesh, India ${ }^{2}$
}

\begin{abstract}
In this paper we analyzed the behavior of the Electromagnetic waves on some of the human tissues. As Electromagnetic waves propagate from one homogeneous medium to another, they experience a change of the wave impedance in the interface. The impedance mismatch generally leads to the reflection, absorption, and transmission of Electromagnetic waves. In the case of non homogeneous media like a human body, it is complex to analyze the behavior of Electromagnetic waves. The reflection and transmission coefficients vary from tissue to tissue and they are dependent on permittivity, conductivity, conductivity and frequency. Moreover, these fundamental parameters are also dependent on frequency. The frequency dependent reflection and transmission coefficients of some human tissues, especially bone tissues are computed at frequency range 0 to $10 \mathrm{GHZ}$ using MATLAB.
\end{abstract}

Keywords: Electromagnetic waves, transmission coefficients, permittivity, conductivity, human tissues

\section{INTRODUCTION}

Bones are the rigid organs that constitute part of the 3. As there are no losses within a perfect conductor, no endo skeleton of vertebrates. It is the basic unit of the energy is absorbed in it

human skeletal system and provides the framework for 4. When an EM wave travelling in one medium is incident and bears the weight of the body, protects the vital organs, upon a second medium, it is partially reflected and supports mechanical movement, hosts hematopoietic cells, partially transmitted.

and maintains iron homeostasis [1]. It is composed of Total fields of a wave at any point after with normal abundant calcium and phosphorus, as well as smaller incidence on a perfect conductor [6]

amounts of bicarbonate, citrate, magnesium, potassium Resultant electric field,

and sodium.Bone tissue can be classified into cortical bone(compact bone): cortical bone consists mainly of haversian systems or secondary osteons.cortical bone forms a relatively thick and dense outer wall and makes up about $80 \%$ of total skeletal mass and cancellous bone (sponge bone) it is located within the medullary cavity [2]. Bone marrow is the flexible tissue in the interior of bones. In humans, red blood cells are produced by cores of bone marrow in the heads of long bones in a process known as hematopoiesis. On average, bone marrow constitutes $4 \%$ of the total body mass of humans, in an adult weighing 65 kilograms, bone marrow typically 2.6 kilograms.It produces approximately 500 billion body cells per day [3].

When an Electromagnetic wave is incident on human tissues some of the energy is transmitted and some is reflected back, because of impedance mismatches. So it is necessary to study the reflection and transmission coefficients of biological tissues. In this unit expressions for and reflection and transmission coefficients are derived from conducting and dielectric media for different incidence cases [4]

$$
\widetilde{\mathrm{E}}_{\mathrm{R}}(\mathrm{z}, \mathrm{t})=2 \mathrm{E}_{\mathrm{i}} \sin \beta \mathrm{z} \sin \omega \mathrm{t}
$$

$E_{i}$ is the amplitude of the electric field of the incident wave, $\mathrm{z}$ is the direction of propagation.

Resultant magnetic field,

$$
\widetilde{\mathrm{H}}_{\mathrm{R}}(\mathrm{z}, \mathrm{t})=2 \mathrm{H}_{\mathrm{i}} \cos \beta \mathrm{z} \cos \omega \mathrm{t}
$$

$\mathrm{H}_{\mathrm{i}}$ is the amplitude of the magnetic field of the incident wave.

Let the electric field of the incident wave be $\mathrm{E}_{\text {incident }}=$ $E_{i} e^{-j \beta z}, \beta=\frac{2 \pi}{\lambda}$

Then the electric field of the reflected wave is

$$
\mathrm{E}_{\text {reflected }}=\mathrm{E}_{\mathrm{r}} \mathrm{e}^{\mathrm{j} \beta \mathrm{z}}
$$

$\mathbf{E}_{\mathbf{i}}=$ Incident field, $\mathbf{E}_{\mathbf{r}}=$ Reflected field.

Reflection Waves on a perfect conductor-normal incidence:

\section{Resultant magnetic field, $\mathbf{H}_{\mathbf{R}}$}

1. When a wave in air is incident on perfect conductor Let us write as $H_{R}$ as normally, it is entirely reflected.

2. As neither $\mathbf{E}$ nor $\mathbf{H}$ can exist in a perfect conductor,

$$
H_{R}(z)=H_{i} e^{-j \beta z}+H_{r} e^{j \beta z}
$$




\section{A. Waves on Dielectric-Normal incidence}

When an EM wave is incident normally on the surface of a dielectric, reflection and transmission takes place.

For a perfect dielectric, $\sigma=0$. Hence, there is no loss or no absorption of energy in it.

The Reflection coefficient is defined as the ratio of reflected wave and incident wave.

That is, the reflection coefficient $=\frac{\text { reflected } \text { wave }}{\text { incident } \text { wave }}$

Reflection coefficient for electric field

$$
E=\dot{\Gamma}_{\mathrm{E}} \equiv \frac{\mathrm{E}_{\mathrm{r}}}{\mathrm{E}_{\mathrm{i}}}
$$

Reflection coefficient for magnetic field

$$
\mathrm{H}=\dot{\Gamma}_{\mathrm{H}} \equiv \frac{\mathrm{H}_{\mathrm{r}}}{\mathrm{H}_{\mathrm{i}}}
$$

Where $\quad E_{r}=$ Reflected electric field

$\mathrm{E}_{\mathrm{i}}=$ Incident electric field

$\mathrm{H}_{\mathrm{r}}=$ reflected magnetic field

$\mathrm{H}_{\mathrm{i}}=$ incident magnetic field

Transmission coefficient is defined as the ratio of transmitted wave and incident wave.

Transmission coefficient $\equiv \frac{\text { transmitted wave }}{\text { incident wave }}$

Transmission coefficient for $\mathrm{E}, \mathrm{T}_{\mathrm{E}} \equiv \frac{\mathrm{E}_{\mathrm{t}}}{\mathrm{E}_{\mathrm{i}}}$

And Transmission coefficient for $\mathrm{H}$ is, $\mathrm{T}_{\mathrm{H}} \equiv \frac{\mathrm{H}_{\mathrm{t}}}{\mathrm{H}_{\mathrm{i}}}$

B. Expressions for reflection and transmission coefficients are [7]:

$$
\begin{gathered}
\dot{\Gamma}_{\mathrm{E}}=\frac{\mathrm{E}_{\mathrm{r}}}{\mathrm{E}_{\mathrm{i}}}=\frac{\eta_{2}-\eta_{1}}{\eta_{2}+\eta_{1}} \\
\dot{\Gamma}_{\mathrm{H}}=\frac{\mathrm{H}_{\mathrm{r}}}{\mathrm{H}_{\mathrm{i}}}=\frac{\eta_{1}-\eta_{2}}{\eta_{1}+\eta_{2}} \\
\mathrm{~T}_{\mathrm{E}}=\frac{\mathrm{E}_{\mathrm{t}}}{\mathrm{E}_{\mathrm{i}}}=\frac{2 \eta_{2}}{\eta_{1}+\eta_{2}} \\
\dot{\Gamma}_{\mathrm{H}}=\frac{\mathrm{H}_{\mathrm{t}}}{\mathrm{H}_{\mathrm{i}}}=\frac{2 \eta_{1}}{\eta+\eta_{2}}
\end{gathered}
$$

Where $\mathrm{n}_{1}$ and $\eta_{2}$ are intrinsic impedances of medium 1 and medium 2 respectively.

\section{OBLIQUE INCIDENCE OF A PLANE WAVE ON DIELECTRIC}

Fig. 2: Incident, Reflected and Transmitted rays

When a wave is incident on a dielectric, a part of it is reflected and a part of it is transmitted through the dielectric. If $\theta_{\mathrm{i}}, \theta_{\mathrm{r}}$ and $\theta_{\mathrm{t}}$ are the angles of the incident, reflected and transmitted rays, $\theta_{\mathrm{i}}=\theta_{\mathrm{r}}$. the angles $\theta_{\mathrm{i}}$ and $\theta_{\mathrm{t}}$ are related by Snell's law, that is,

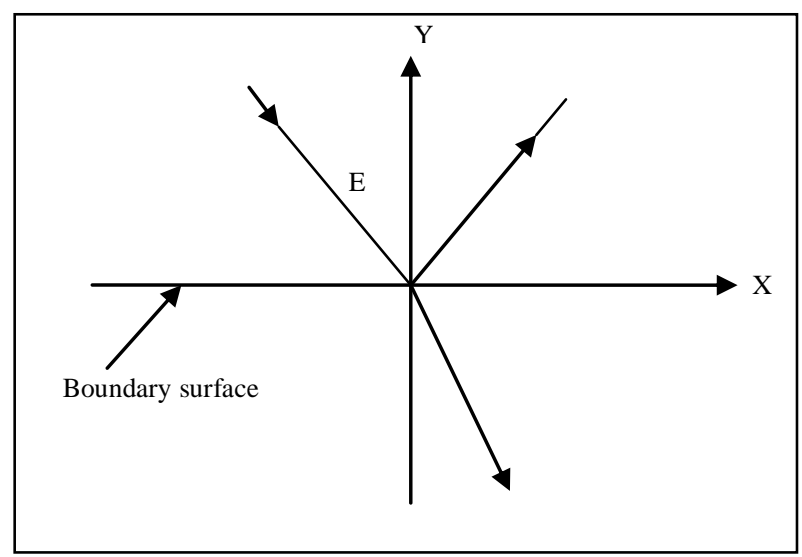

Fig. 1: Incident, Reflected and Transmitted rays

From the above the reflection coefficient is

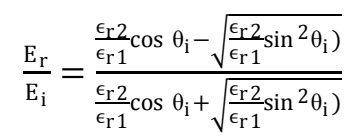

\section{PERPENDICULAR POLARISATION}

Consider Fig. 5.4 in which $\mathrm{E}$ is $\mathrm{z}$-directed and $\mathrm{x}-\mathrm{y}$ is the plane of incidence.

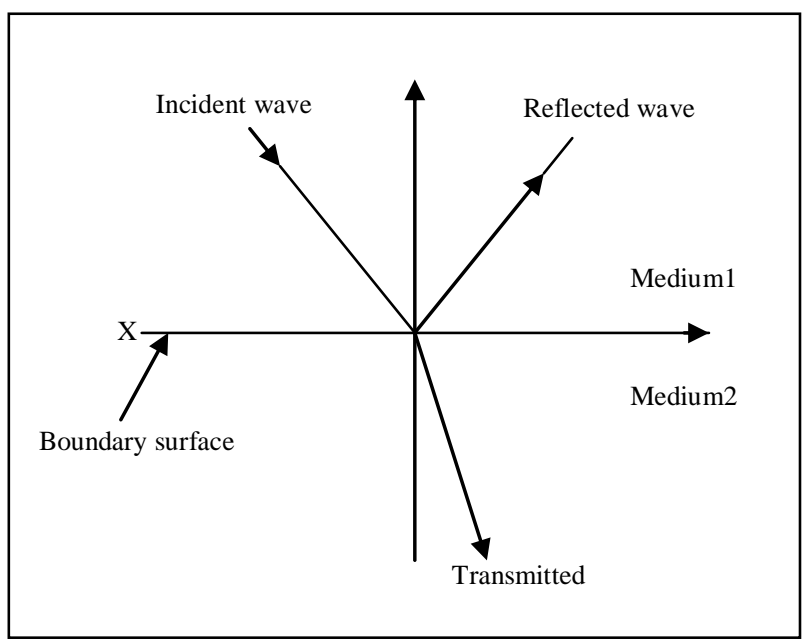

Fig. 2: Incident, Reflected and Transmitted rays

From the boundary conditions and further simplification we get

$$
\frac{\mathrm{E}_{\mathrm{r}}}{\mathrm{E}_{\mathrm{i}}}=\frac{\cos \theta_{\mathrm{i}}-\sqrt{\left.\left(\frac{\epsilon_{\mathrm{r} 2}}{\epsilon_{\mathrm{r} 1}}\right)-\sin ^{2} \theta_{\mathrm{i}}\right)}}{\cos \theta_{\mathrm{i}}+\sqrt{\left.\left(\frac{\epsilon_{\mathrm{r} 2}}{\epsilon_{\mathrm{r} 1}}\right)-\sin ^{2} \theta_{\mathrm{i}}\right)}}
$$

\section{ELECTROMAGNETIC WAVES WITH BIOLOGICAL TISSUES}

When a beam of EM waves strikes the surface of a tissue, the EM energy interacts with the atoms and molecules of the tissue. The EM energy may be transmitted, absorbed, scattered or reflected, or it can excite fluorescence 
depending upon the properties of the substance. The interaction, however, does not involve in a permanent transfer of energy.

The velocity at which EM energy is propagated through a medium is less than its velocity in a vacuum. It depends upon the kind and concentration of atoms, ions or molecules present in the medium. Figure 5.5 shows various possibilities which might result when a beam of radiation strikes the substance. These are:

(a).The EM energy may be transmitted with little absorption taking place, and therefore, without much energy loss.

(b).The direction of propagation of the beam may be altered by reflection, refraction, diffraction or scattering.

(c). The EM energy may be absorbed in part or completely by the tissue.

In absorption spectrophotometry, we are usually concerned with absorption and transmission. Generally, the conditions under which the sample is examined are such that they keep reflection and scattering to a minimum

Absorption spectrophotometry is based on the principles that the amount of absorption that occurs is dependent on the number of molecules present in the number of molecules present in the absorbing material. Therefore, the intensity of the EM energy leaving the tissue may be used as an indication of the concentration of the material [5].

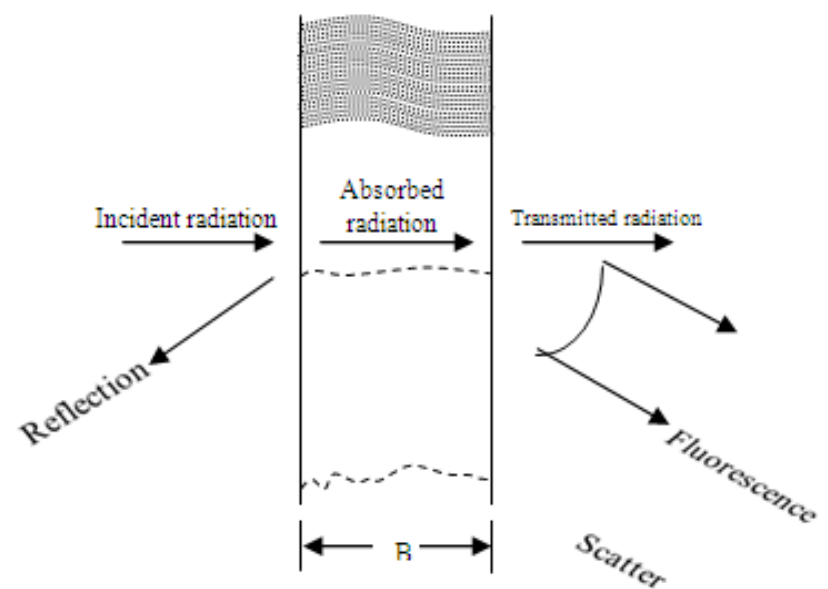

Fig. 3: Interaction of EM energy with tissue.

The reflection and transmission of ultrawideband electromagnetic pulses through multi-layered biological media consisting of three biological tissue layers representing skin, fat and muscle was analyzed by C.L.Palombini and K.E.Oughstuny.The frequency dependent reflection and transmission coefficients of the multilayer stack were computed using the transfer matrix method. Here in this paper, we analyze the reflection and transmission coefficients of Electromagnetic waves through the bone tissues using MATLAB.

\section{RESULTS}

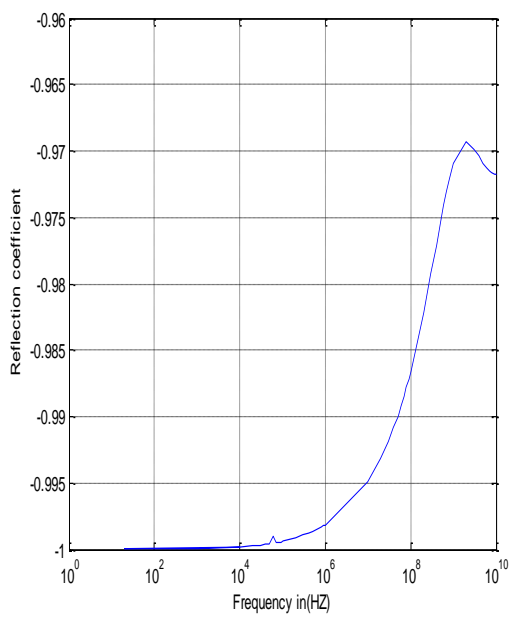

Fig.4: Reflection coefficient of Bone cancels/Blood vs. Frequency

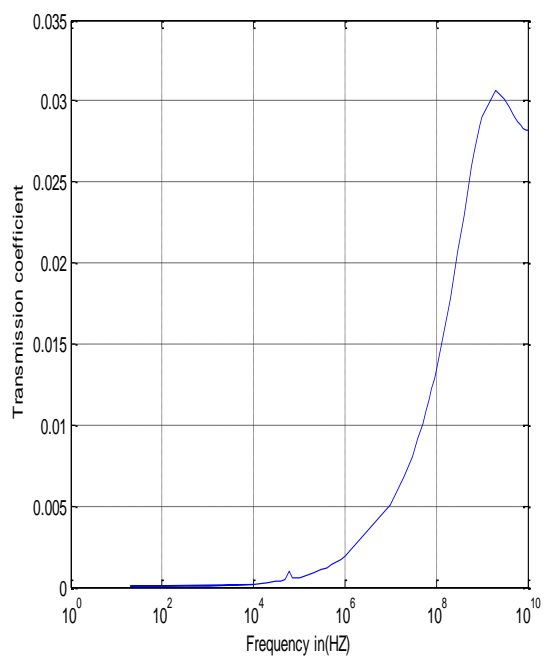

Fig.5: Transmission coefficient of Bone cancellous/Blood vs. Frequency

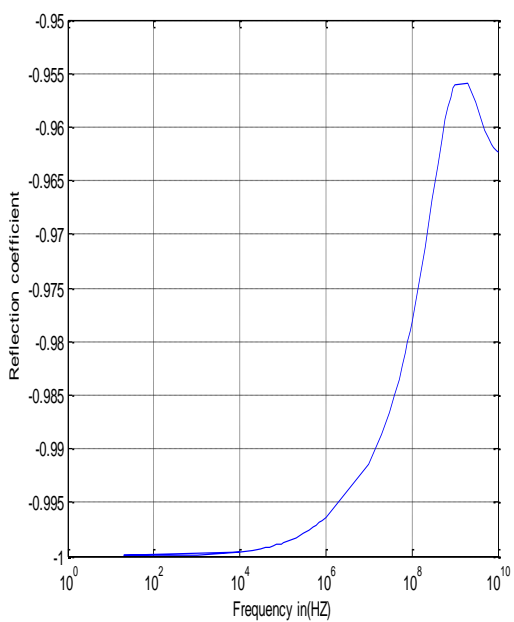

Fig.6: Reflection coefficient of Bone cortical/Blood vs. Frequency 


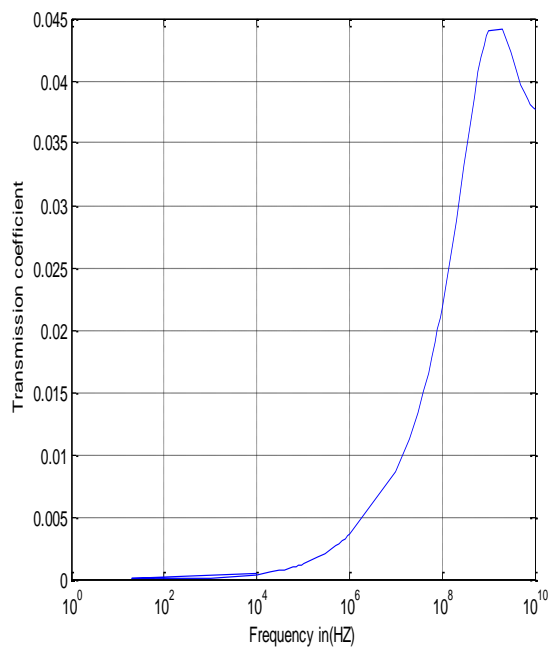

Fig.7: Transmission coefficient of Bone cortical/Blood vs. Frequency

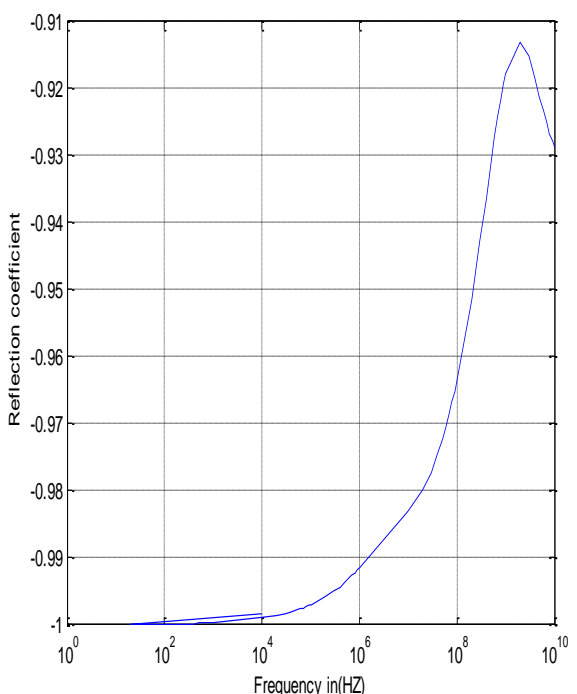

Fig.8: Reflection coefficient of Bone marrow /Blood vs. Frequency

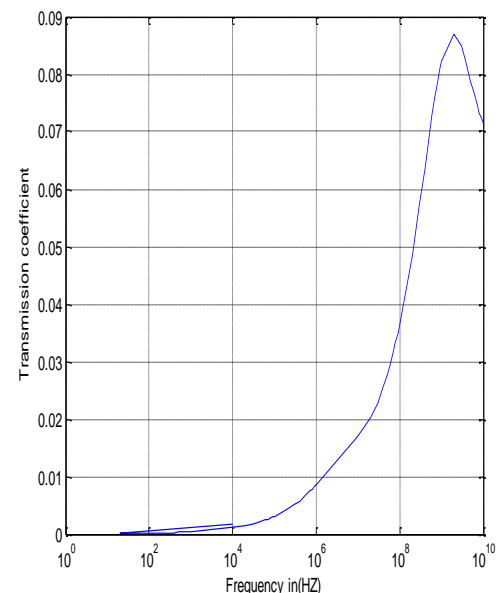

Fig.9: Transmission coefficient of Bone marrow /Blood vs. Frequency

\section{CONCLUSION}

In this paper, we have analyzed the reflection and transmission coefficients for different parts of bone with the interface of blood. The different parts of the bone those we have considered are bone cancellous, bone corticle and bone marrow. It is evident from the results the transmission and reflection coefficient for bone and blood interface exhibits overshoot that approximate about $1 \mathrm{GHz}$. From the results it is observed that the Reflection coefficient of all bone adjacent with blood tissues value is ' -1 ' at very low frequencies. The transmission coefficient value is ' 0 ' from very low frequencies to $10 \mathrm{KHz}$ frequency. The highest reflection and transmission coefficients observed from $1 \mathrm{GHz}$ to $5 \mathrm{GHz}$ microwave frequency range. Further, the reflection and transmission coefficients decreased at $10 \mathrm{GHz}$ frequency for all bone tissues interfacing with blood.

\section{REFERENCES}

[1] MC carthy EF,frassica FJ,Anatomy and physiology of bone. Pathology of bone and joint disorders Philadelphia,pa:WB saunder; 1998:25-50

[2] Junqueria LC,carneiro,long JA Bone. Basic histology. $5^{\text {th }}$ ed. norwalk,conn:Appleton century-crofts;1986:140-65

[3] Vunjak. Novakovic ,G; tendon,[2010]"challenges in cardiac tissue engineering", tissue engineering part B.

[4] C.L. Palombini, K. E. Oughstun "Reflection and Transmission of pulsed Electromagnetic fields through Multilayered biological Media" 978-1-61284-978-2/11 IEEE 2011.

[5] H.C. Kim, J. P. Verboncoeur "Reflection, Absorption and transmission of TE electromagnetic Wave propagation in a nonuniform plasma slab", Elsevier, 31 January 2007.

[6] B. R. Chawla and Hillel Unz "Reflection and Transmission of Electromagnetic Waves Normally Incident on a Plasma Slab Moving Uniformly Along a Magnetostatic Field" IEEE Transactions on Antennas and propagation, Vol-no. 6 November 1969.

[7] Y. Ratna Kumar and Prof. G.S.N. Raju, "Reflection and Transmission Coefficients of Different Biological Tissues", Vol. 2, Issue 8, pp. 5509-5514, August 2014

\section{BIOGRAPIES}

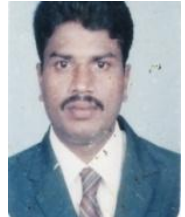

Dr.Y. Ratna Kumar did his B. Pharmacy in JNTUH and $M$. Tech (Biomedical Engg.) in Andhra University. At present, he is Assistant Professor (c) in the Centre for BioMedical Engineering, Dept. of Electronics and Communication Engineering, AU College of Engineering (A), Visakhapatnam, and Ph.D. under the guidance of Prof. G.S.N. Raju. He has published 15 International Journals/ Conferences. He is a life member of the PCI, SEMCE (I), BMSI, and ISB.

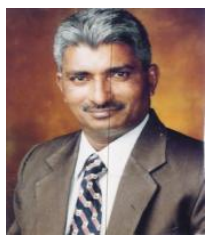

Dr. G.S.N. Raju received his B.E., M.E. With distinction and first rank from Andhra University, Visakhapatnam and Ph.D. from IIT, Kharagpur. $\mathrm{He}$ is Professor of Electronics and Communication Engineering in Andhra University College of Engineering, Visakhapatnam, India. He is in teaching and research for the last 30 years, Andhra University, Visakhapatnam. He guided 16 Ph.D.s in the fields of Antennas, Electromagnetics, EMI/EMC and Microwave, Radar Communications, Electronic circuits. Prof. Raju has published about 260 technical papers in National/ International Journals/Conference 
Journals and transactions. He is the recipient of "The State Best Teacher Award" from the Government of Andhra Pradesh in 1999, "The Best Researcher Award" in 1994, Prof.Aiya Memorial "National IETE Award" for his best Research guidance in 2008 and "Dr. Sarvepalli Radha Krishnan Award for the Best Academician of the year 2007". He was a visiting Professor in the University of Paderborn and also in the University Karlsruhe, Germany in 1994. At present he holds the positions of V,C, Andhra University, Visakhapatnam, Chief Editor of National Journal of Electromagnetic Compatibility, India. Prof. Raju has published five textbooks "Antennas and Wave Propagation", "Electromagnetic Field Theory and Transmission Lines", "Electronics Devices and Circuits", "Microwave Engineering", Radar Engineering and Navigational Aids".Prof. Raju has been the best faculty performer in Andhra University with the performance index of $99.37 \%$. 